\title{
In vitro antimicrobial susceptibility testing of human Brucella melitensis isolates from Qatar between 2014 - 2015
}

\author{
Anand Deshmukh ${ }^{1 *}$, Ferry Hagen², Ola Al Sharabasi ${ }^{1}$, Mariamma Abraham', Godwin Wilson ${ }^{3}$, Sanjay Doiphode ${ }^{1}$
} Muna Al Maslamani $i^{4}$ and Jacques F Meis 2,5

\begin{abstract}
Background: Brucellosis is one of the most common zoonotic disease affecting humans and animals and is endemic in many parts of the world including the Gulf Cooperation Council region (GCC). The aim of this study was to identify the species and determine the antimicrobial susceptibility pattern of Brucella strains isolated from clinical specimens, from Qatar.

Results: We evaluated 231 Brucella isolates. All isolates were identified as B. melitensis. All the isolates were susceptible to doxycycline, tetracycline, streptomycin, gentamicin, trimethoprim / sulfamethoxazole and ciprofloxacin except rifampicin, where $48 \%$ of the strains showed elevated MICs (>1 mg/L). The rifampicin-resistance related hotspots within the rpoB gene were amplified and sequenced using PCR and no rpoB mutations were found in strains with rifampicin MICs of $>2 \mathrm{mg} / \mathrm{L}$.
\end{abstract}

Conclusion: This study identified B. melitensis as the etiological agent of brucellosis in Qatar. No resistant isolates were detected among conventionally used antimicrobial agents.

Keywords: Brucella melitensis, Brucellosis, Antimicrobial susceptibility testing, Qatar

\section{Background}

Brucellosis is a worldwide zoonotic disease in both animals and humans with an estimated 500,000 new cases annually [1]. Although brucellosis is a notifiable disease in many countries, it is probably underreported and official numbers do not reflect the true incidence of this infection [2]. Thus the true incidence of human brucellosis is unknown and the estimated burden of the disease varies widely, from $<0.03$ to $>160$ per 100,000 population. The highest recorded incidence of human brucellosis occurs in the Middle East and Central Asia [3]. Brucellosis is transmitted to humans by direct contact with infected animals or consumption of unpasteurized or inadequately cooked milk or milk products, inhalation of infected aerosolized particles and, to a lesser extent, meat derived from cattle, sheep, goats, pigs, camels, yaks, buffaloes or dogs. Four species of the genus Brucella are usually pathogenic for

\footnotetext{
* Correspondence: adeshmukh@hamad.qa

'Microbiology Division, Hamad General Hospital, Doha, Qatar

Full list of author information is available at the end of the article
}

humans, which include B. melitensis (from sheep/goats and camel), B. abortus (from cattle and other bovine animals), B. suis (from pigs), and B. canis (from dogs) [4]. Routine susceptibility testing of Brucella species is not performed due to the need for containment level 3 facilities [4] and concerns of laboratory-acquired infections [5]. The main objective of this study was to determine the antimicrobial susceptibility pattern of human Brucella isolates from patients in Qatar collected over a 10 -year period.

\section{Methods}

Bacterial isolates

A total of 231 strains of Brucella isolates obtained from various clinical specimens (blood $n=225$ and synovial fluid $\mathrm{n}=6$ ) collected between January 2005 and July 2014 from patients attending Hamad General Hospital and $\mathrm{Al}$ Khor Hospital, Qatar, were included in the study. All the isolates were collected as a part of standard patient care. Identification of isolates was based on colony 
morphology, Gram stain, oxidase, catalase, Vitek 2 compact (bioMerieux, Durham, USA) and Maldi-TOF MS (Bruker Daltonics, Bremen, Germany). For Maldi-TOF MS, cultures were processed using the ethanol/formic acid/acetonitrile protocol [6]. All cultures were processed in a Class II biological safety cabinet in a negative pressure room.

\section{Antimicrobial susceptibility testing}

Susceptibility of seven antibiotics (doxycycline, tetracycline, streptomycin, gentamicin, rifampicin, trimethoprim/ sulfamethoxazole and ciprofloxacin) was determined with a gradient strip method (E-test strips, bioMerieux, Marcy L'Etoile, France). The antimicrobial strips were placed on Muller Hinton blood agar according to the manufacturer's guidelines and read after $48 \mathrm{~h}$ incubation in ambient air at $37{ }^{\circ} \mathrm{C}$. Minimum inhibitory concentrations (MIC) breakpoints of streptomycin, gentamicin, tetracycline, doxycycline and trimethoprim-sulfamethoxazole were used as recommended by CLSI [7]. As MIC breakpoints for Brucella against rifampicin and ciprofloxacin have not been established, guidelines for slow-growing bacteria (H.influenzae) were used [8, 9]. The MIC was interpreted as the value at which the inhibition zone intercepted the scale on the E-test strip and the MIC values were rounded up (to the next higher dilution) with the corresponding MIC of the microbroth dilution method. $\mathrm{MIC}_{50}$ and $\mathrm{MIC}_{90}$ levels were defined as the lowest concentration of the antibiotic at which $50 \%$ and $90 \%$ of the isolates were inhibited, respectively.

The reference strains- H. influenza ATCC 10211, E.coli ATCC 25922, S.pneumoniae ATCC 49619 and S.aureus ATCC 29213 were used as quality control.

\section{PCR assay of $r p o B$ gene}

For isolates $(n=17)$ that showed a rifampicin MIC of $>$ $2 \mathrm{mg} / \mathrm{L}$, the rifampicin-resistance related hotspots within the $r p o B$ gene were amplified and sequenced as described before [10]. The sequences were compared to rроB sequences of wildtype and resistant isolates as provided by other studies [10, 11].

\section{Results}

All the isolates were identified by Vitek II compact and Maldi-TOF as B. melitensis. Table 1 represents MIC range; $\mathrm{MIC}_{50}$ and $\mathrm{MIC}_{90}$ of each antibiotic tested and MIC distributions are shown in Fig. 1.

All the isolates were susceptible to doxycycline $\left(\mathrm{MIC}_{90}\right.$, $0.25 \mathrm{mg} / \mathrm{L})$, gentamicin $\left(\mathrm{MIC}_{90}, 0.5 \mathrm{mg} / \mathrm{L}\right)$, streptomycin $\left(\mathrm{MIC}_{90}, 2 \mathrm{mg} / \mathrm{L}\right)$, trimethoprim-sulfamethoxazole $\left(\mathrm{MIC}_{90}\right.$, $0.25 \mathrm{mg} / \mathrm{L})$ and tetracycline $\left(\mathrm{MIC}_{90}, 0.25 \mathrm{mg} / \mathrm{L}\right)$. In addition, ciprofloxacin $\left(\mathrm{MIC}_{90}, 0.5 \mathrm{mg} / \mathrm{L}\right)$ showed $100 \%$ susceptibility when MIC breakpoint criteria for H.influenzae [8] were used. For rifampicin, the MIC values ranged from 0.008 to $4 \mathrm{mg} / \mathrm{L}$ and when compared with MIC breakpoint criteria for H.influenzae [8], resistance (MIC $\geq$ $2 \mathrm{mg} / \mathrm{L}$ ) was demonstrated in $48 \%$ strains. After sequencing of the rpoB gene, no mutations were found in isolates with $\mathrm{MIC}$ of $>2 \mathrm{mg} / \mathrm{L}(\mathrm{n}=17)$.

\section{Discussion}

Brucella melitensis is the commonest etiological agent causing human brucellosis worldwide. Intracellular localization of Brucella species within monocytes and macrophages of the reticulo-endothelial system limits the choices of antimicrobial agents for effective treatment of systemic and localized brucellosis. Although a variety of antimicrobial agents appear to be active in vitro, the results of susceptibility testing do not always correlate with clinical efficacy [4].

The recommended method for antimicrobial susceptibility testing of Brucella species is microbroth dilution using unsupplemented Brucella broth [7]. When the Etest method was compared with microbroth dilution, Gur et al. found no significant variations in obtained MIC values [12]. This method appear to be reliable, reproducible, easily performed, and produces similar results to those of conventional methods for Brucella on Muller Hinton blood agar [13, 14] and it is therefore that we used E-test for susceptibility testing.

Amongst tetracyclines, doxycycline is the most common antimicrobial drug used for treatment of uncomplicated brucellosis in adults and children of more than 8 years [4]. In the present study, MICs of tetracycline

Table 1 Antimicrobial susceptibility of B.melitensis

\begin{tabular}{lllll}
\hline & Range $(\mathrm{mg} / \mathrm{L})$ & $\mathrm{MIC}_{50}(\mathrm{mg} / \mathrm{L})$ & $\mathrm{MIC}_{90}(\mathrm{mg} / \mathrm{L})$ & Breakpoints for susceptibility $(\mathrm{mg} / \mathrm{L})$ \\
\hline Doxycycline & $0.032-1$ & 0.125 & 0.25 & $\leq 1[7]$ \\
Tetracycline & $0.032-0.5$ & 0.125 & 0.25 & $\leq 1[7]$ \\
Gentamicin & $0.064-2$ & 0.25 & 0.5 & $\leq 4[7]$ \\
Streptomycin & $0.125-4$ & 0.5 & 2 & $\leq 8[7]$ \\
Trimethoprim-Sulfamethoxazole & $0.008-2$ & 0.064 & 0.25 & $\leq 2 / 38[7]$ \\
Rifampicin & $0.008-4$ & 1 & 2 & $\leq 1[8]$ \\
Ciprofloxacin & $0.064-0.5$ & 0.25 & 0.5 & $\leq 1[8]$ \\
\hline
\end{tabular}




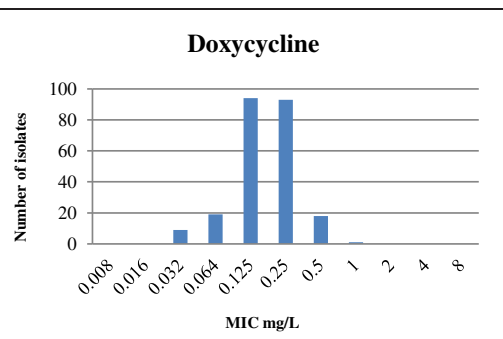

Gentamicin

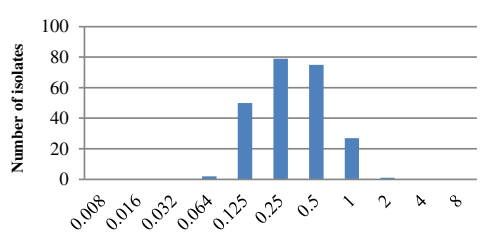

MIC mg/L

Trimethoprim- sulfamethoxazole

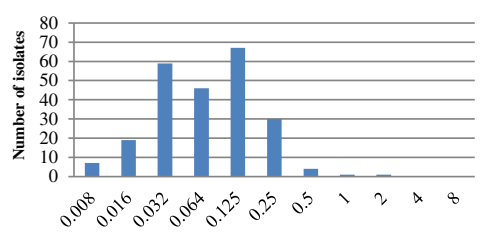

MIC mg/L

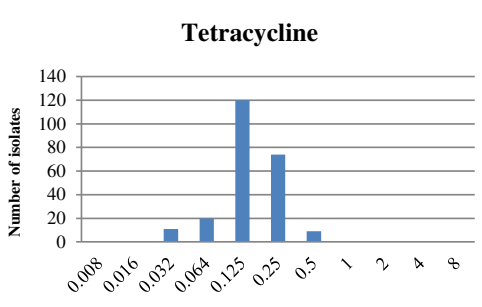

MIC mg/L

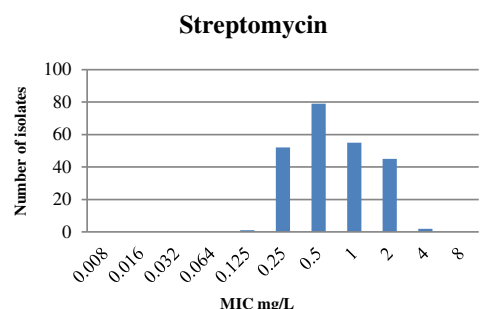

MIC mg/L

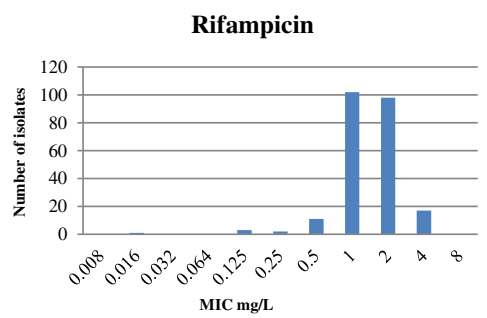

MIC mg/L

Ciprofloxacin

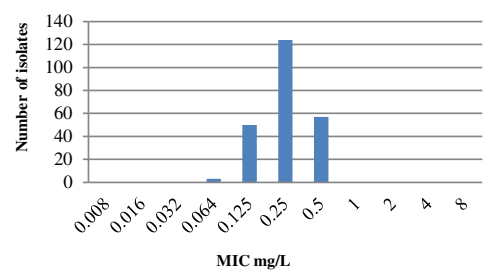

Fig. 1 Distribution of MIC values in indicated antimicrobials of B. melitensis from Qatar

(range - 0.032-0.5 $\mathrm{mg} / \mathrm{L}$ ) and doxycycline (range - 0.032$1 \mathrm{mg} / \mathrm{L}$ ) are found to be in the susceptible category and $\mathrm{MIC}_{50}$ and $\mathrm{MIC}_{90}$ values are consistent with previous reports $[9,13,15,16]$. However, increased MICs of doxycycline have been reported in a study from Mexico [17].

The World Health Organization (WHO) recommends doxycycline (6 weeks) in combination with aminoglycosides (streptomycin or gentamicin for 2-3 weeks) or rifampicin (6 weeks) [4]. The most preferred and effective aminoglycoside in treatment of brucellosis is streptomycin $[4,18,19]$. Combination with streptomycin was found to be superior as compared to rifampicin, in terms of relapse or treatment failure [18]. Even though gentamicin is more active in vitro and is associated with fewer side effects than streptomycin, there are not enough studies, which justify the replacement of streptomycin $[4,19]$. In the present study, all the strains were susceptible to streptomycin $(0.125-4 \mathrm{mg} / \mathrm{L})$ and gentamicin $(0.064-2 \mathrm{mg} / \mathrm{L})$ and found to be in the range described previously $[9,15]$. Nevertheless, a high $\mathrm{MIC}_{90}$ of streptomycin $(8 \mathrm{mg} / \mathrm{L})$ was reported from Turkey [16].

Another commonly used drug in treatment of brucellosis in combination with doxycycline is rifampicin. In spite of the proven efficacy with aminoglycoside combination, due to the need for parenteral administration, convenience and poor patient compliance, healthcare professionals prefer the all-oral regimen of doxycycline/ rifampicin [18, 20]. A meta-analysis on various combinations used for the treatment of brucellosis, has shown higher relapse rate with doxycycline/rifampicin when compared to doxycycline/streptomycin regimen [21]. It has been suggested that rifampicin may enhance the plasma clearance of doxycycline resulting in lower doxycyline levels [22] and the possibility that its use could contribute to emergence of rifampicin- resistant Mycobacterium tuberculosis in endemic countries [23]. For in-vitro susceptibility testing, MIC breakpoints of rifampicin against Brucella has not been established and therefore these organisms cannot 
be confidently characterised as susceptible, intermediate or resistant. However, when compared with CLSI reference values for H.influenzae [8], in the present study, $48 \%$ of Brucella strains showed elevated MIC's of rifampicin (>1 mg/L). Higher MIC's to rifampicin has been reported previously from Egypt (64 \%) [9] and Malaysia (70 \%) [15]. However, the impact of high MIC's on clinical outcome in these countries is not known. In Qatar, in a retrospective cohort study, combination of doxycyline with streptomycin was found to be the preferred regimen followed by doxycyline with rifampicin and no relapse or therapeutic failures were detected [24].

Mutations conferring rifampin resistance are confined almost exclusively to the $r p o B$ gene in most organisms and result in a decreased affinity of the DNA-dependent RNA polymerase to the antibiotic. Alternative mechanisms of rifampin resistance also have been observed [25]. Along with mutations in the rpoB gene, excitation of several metabolic processes may also be a contributing factor in conferring rifampicin resistant in Brucella [26]. We did not find any mutations in the $r p o B$ gene in isolates with elevated MIC's in this study, findings consistent with previous studies [14, 27, 11]. However, further research is required to find possible other mechanisms of rifampicin resistance in Brucella.

Trimethoprim/sulfamethoxazole (TMP/SMX) is an alternative agent recommended for treatment of brucellosis in pregnancy and in children under 8 years old. It has also been recommended for treatment of osteoarticular complications, neurobrucellosis and endocarditis, in combination with doxycycline, aminoglycoside and rifampicin [4]. TMP/SMX, when used in combination with rifampicin, was found to be effective in pediatric patients (8 weeks regimen). High relapse rates were noted when TMP/SMX was used as a monotherapy in pediatric brucellosis [28]. Development of resistance to TMP/SMX to B. melitensis is an important issue. High rate of resistance to TMP/SMX has been reported from previous studies $[17,29]$. In the present study, TMP/SMX was found to be susceptible with low MIC 50 $(0.064 \mathrm{mg} / \mathrm{L})$ and $\mathrm{MIC}_{90}(0.25 \mathrm{mg} / \mathrm{L})$ when compared to other antimicrobials which is consistent with previous reports $[9,13,15]$.

Ciprofloxacin is a potential alternative therapeutic option in the treatment of brucellosis with excellent oral bioavailability and reaching high concentrations in phagocytic cells [30]. However, ciprofloxacin is not recommended for monotherapy due to the lack of bactericidal activity at the intracellular acidic $\mathrm{pH}$ [31], high rate of relapse and the risk of development of overall fluoroquinolone resistance in the community [32]. Therefore, its use in combination with doxycycline is recommended as an acceptable alternative but not as first line regimen $[29,33]$. We found ciprofloxacin MICs $(0.064-0.5 \mathrm{mg} / \mathrm{L})$ in the susceptible range when compared to MIC breakpoint criteria of slow growing bacteria and these findings are consistent with previous studies $[9,16]$. Ciprofloxacin based therapy might play a role as an alternative regimen especially in patients with intolerance to commonly used drugs and relapsed disease [30].

\section{Conclusion}

In summary, this study identified B. melitensis as the etiological agent of brucellosis in Qatar. No resistant isolates were detected among conventional antimicrobial agents. Periodic monitoring of antimicrobial resistance in the Middle East, especially in the Gulf Cooperating Council (GCC) in light of the emerging resistance and development of CLSI or EUCAST interpretive criteria for rifampicin are needed.

\section{Competing interests}

The authors declare that they have no competing interests.

\section{Authors' contributions}

$\mathrm{AD}$ participated in the design of the study, coordinated all work related to the study, performed data analysis and drafted the manuscript; FH did the $r p o B$ PCR and participated in data analysis; OAS and MA executed the work related to identification of Brucella and antimicrobial susceptibility test; GW, SD and MAM participated in the design of the study; JFM participated in the design of the study, performed data analysis and critically reviewed the manuscript. All authors read and approved the final manuscript.

\section{Acknowledgements}

Funding- this research project was supported by NPRP grant 5-746-3-176 from Qatar National Research Foundation (QNRF). The content and statement made herein are solely the responsibility of the authors.

The authors would like to thank the Bacterial Special Pathogen Branch at Center for Disease Control and Prevention, USA, for providing the Brucella Maldi-TOF database.

\section{Author details}

${ }^{1}$ Microbiology Division, Hamad General Hospital, Doha, Qatar. ${ }^{2}$ Department of Medical Microbiology and Infectious Diseases, Canisius-Wilhelmina Hospital, Nijmegen, The Netherlands. ${ }^{3}$ Microbiology Division, Al Khor Hospital, Doha, Qatar. ${ }^{4}$ Department of Infectious Diseases, Hamad General Hospital, Doha, Qatar. ${ }^{5}$ Department of Medical Microbiology, Radboud University Medical Center, Nijmegen, The Netherlands.

Received: 3 December 2014 Accepted: 1 June 2015 Published online: 16 June 2015

\section{References}

1. World Health Organization (WHO). Fact sheet N173. Geneva, Switzerland: WHO; 1997.

2. Seleem MN, Boyle SM, Sriranganathan N. Brucellosis: a re-emerging zoonosis. Vet Microbiol. 2010;140:392-8.

3. Pappas G, Papadimitriou P, Akritidis N, Christou L, Tsianos EV. The new global map of human brucellosis. Lancet Infect Dis. 2006;6:91-9.

4. Corbel MJ. Brucellosis in humans and animals. Geneva (Switzerland): World Health Organization; 2006.

5. Robichaud S, Libman M, Behr M, Rubin E. Prevention of laboratory-acquired brucellosis. Clin Infect Dis. 2004;38:e119-22.

6. Lista F, Reubsaet FA, De Santis R, Parchen RR, de Jong AL, Kieboom J, et al. Reliable identification at the species level of Brucella isolates with MALDITOF-MS. BMC Microbiol. 2011;11:267.

7. CLSI. Methods of antimicrobial dilution and disk susceptibility testing of infrequently isolated or fastidious bacteria. Approved guidelines- second edition. CLSI document M45-A2. Wayne, PA: Clinical and Laboratory Standard Institute; 2010. 
8. CLSI. Performance standard for antimicrobial susceptibility testing: twentyfourth informational supplement. CLSI document M100-S24. Wayne, PA: Clinical and Laboratory Standard Institute; 2014.

9. Abdel-Maksoud M, House B, Wasfy M, Abdel-Rahman B, Pimentel G, Roushdy $\mathrm{G}$, et al. In vitro antibiotic susceptibility testing of Brucella isolates from Egypt between 1999 and 2007 and evidence of probable rifampin resistance. Ann Clin Microbiol Antimicrob. 2012;11:24.

10. Marianelli C, Ciuchini F, Tarantino M, Pasquali P, Adone R. Genetic bases of the rifampicin resistance phenotype in Brucella spp. J Clin Microbiol. 2004:42:5439-43.

11. Valdezate S, Navarro A, Medina-Pascual MJ, Carrasco G, Saéz-Nieto JA. Molecular screening for rifampicin and fluoroquinolone resistance in a clinical population of Brucella melitensis. I Antimicrob Chemother. 2010;65:51-3

12. Gür D, Kocagöz S, Akova M, Unal S. Comparison of E test to microdilution for determining in vitro activities of antibiotics against Brucella melitensis. Antimicrob Agents Chemother. 1999:43:2337.

13. Bayram Y, Korkoca H, Aypak C, Parlak M, Cikman A, Kilic S, et al. Antimicrobial susceptibilities of Brucella isolates from various clinical specimens. Int J Med Sci. 2011:8:198-202.

14. Marianelli C, Graziani C, Santangelo C, Xibilia MT, Imbriani A, Amato R, et al. Molecular epidemiological and antibiotic susceptibility characterization of Brucella isolates from humans in Sicily, Italy. J Clin Microbiol. 2007;45:2923-8.

15. Hashim R, Ahmad N, Mohamed Zahidi J, Tay BY, Mohd Noor A, Zainal S, et al. Identification and in vitro antimicrobial susceptibility of Brucella species isolated from human brucellosis. Int J Microbiol. 2014;2014:596245.

16. Kaya O, Akçam FZ, Yaylı G. Investigation of the in vitro activities of various antibiotics against Brucella melitensis strains. Turk J Med Sci. 2012;42:145-8.

17. López-merino A, Contreras-rodríguez A, Migranas-ortiz R, Orrantia-gradín R, Hernández-oliva GM, Torres Y, et al. Susceptibility of Mexican Brucella isolates to moxifloxacin, ciprofloxacin and other antimicrobials used in the treatment of human brucellosis. Scand J Infect Dis. 2004;36:636-8.

18. Solís García del Pozo J, Solera J. Systematic review and meta-analysis of randomized clinical trials in the treatment of human brucellosis. PLoS One. 2012;7:e32090

19. Ariza J, Bosilkovski M, Cascio A, Colmenero JD, Corbel MJ, Falagas ME, et al. Perspectives for the treatment of brucellosis in the 21st century: the loannina recommendations. PLoS Med. 2007;4:e317.

20. Pappas G, Siozopoulou V, Akritidis N, Falagas ME. Doxycycline-rifampicin: physicians' inferior choice in brucellosis or how convenience reigns over science. J Infect. 2007:54:459-62.

21. Skalsky K, Yahav D, Bishara J, Pitlik S, Leibovici L, Paul M. Treatment of human brucellosis: systematic review and meta-analysis of randomised controlled trials. BMJ. 2008;336:701-4.

22. Colmenero JD, Fernandez-Gallardo LC, Agundez JA, Sedeno J, Benitez J, Valverde E. Possible implications of doxycycline-rifampin interaction for treatment of brucellosis. Antimicrob Agents Chemother. 1994;38:2798-802.

23. Grayson ML, Kucers A. Kucers' the use of antibiotics a clinical review of antibacterial, antifungal, antiparasitic, and antiviral drugs. 6th ed. London: Hodder Arnold; 2010.

24. Rahil Al, Othman M, Ibrahim W, Mohamed MY. Brucellosis in Qatar: a retrospective cohort study. Qatar Med J. 2014;1:25.

25. Goldstein BP. Resistance to rifampicin: a review. J Antibiot (Tokyo). 2014:67:625-30.

26. Sandalakis V, Psaroulaki A, De Bock PJ, Christidou A, Gevaert K, Tsiotis G, et al. Investigation of rifampicin resistance mechanisms in Brucella abortus using MS-driven comparative proteomics. J Proteome Res. 2012;11:2374-85.

27. Sayan M, Kilic S, Uyanik MH. Epidemiological survey of rifampicin resistance in clinic isolates of Brucella melitensis obtained from all regions of Turkey. J Infect Chemother. 2012;18:41-6.

28. Lubani MM, Dudin Kl, Sharda DC, Ndhar DS, Araj GF, Hafez HA, et al. A multicenter therapeutic study of 1100 children with brucellosis. Pediatr Infect Dis J. 1989:8:75-8.

29. Kinsara A, Al-Mowallad A, Osoba AO. Increasing resistance of Brucellae to co-trimoxazole. Antimicrob Agents Chemother. 1999;43:1531.

30. Easmon CS, Crane JP, Blowers A. Effect of ciprofloxacin on intracellular organisms: in-vitro and in-vivo studies. J Antimicrob Chemother 1986;18(Suppl D):43-8.
31. Akova M, Gur D, Livermore DM, Kocagoz T, Akalin HE. In vitro activities of antibiotics alone and in combination against Brucella melitensis at neutral and acidic pHs. Antimicrob Agents Chemother. 1999;43:1298-300.

32. Pappas G, Christou L, Akritidis N, Tsianos EV. Quinolones for brucellosis: treating old diseases with new drugs. Clin Microbiol Infect. 2006;12:823-5.

33. Falagas ME, Bliziotis IA. Quinolones for treatment of human brucellosis: critical review of the evidence from microbiological and clinical studies. Antimicrob Agents Chemother. 2006;50:22-33.

\section{Submit your next manuscript to BioMed Central and take full advantage of:}

- Convenient online submission

- Thorough peer review

- No space constraints or color figure charges

- Immediate publication on acceptance

- Inclusion in PubMed, CAS, Scopus and Google Scholar

- Research which is freely available for redistribution 